\title{
Réplica a la carta al editor Aclaración sobre educación digital en el pregrado de medicina
}

\author{
Reply to the letter Clarification about digital education in undergraduate medical education
}

\section{Señor Editor:}

En respuesta a la carta al Editor "Aclaración sobre educación digital en el pregrado de medicina", nos gustaría responder a la misma desde la humildad y de la aceptación de algunas de sus observaciones:

Con respecto a la función de chat para mensajería instantánea de la plataforma EVA, fue una función que no consideramos debido a que, en nuestra experiencia, no tuvo un uso muy frecuente. En su lugar, y hasta el momento, se prefiere utilizar otras vías como aplicativos de mensajería o llamada directa para la comunicación sincrónica con los docentes u otros estudiantes de la carrera.

Con respecto al uso de foros, si bien el uso de foros brinda una posibilidad para la comunicación sincrónica, en nuestro conocimiento, tampoco era muy utilizado y, por otro lado, actualmente se ha empezado a utilizar la plataforma Blackboard en lugar del EVA ${ }^{(1)}$. Consideramos que sería oportuna la realización de una encuesta periódica dirigida a estudiantes y docentes acerca del conocimiento, frecuencia y dificultades en el uso de las distintas funciones de estas plataformas con la finalidad de poder identificar en qué aspectos se podrían realizar capacitaciones dirigidas y así tener un mejor aprovechamiento de las bondades que estos ofrecen para la educación.

Con relación al uso de un segundo servidor para las evaluaciones, si bien la propuesta de un segundo servidor no sería ventajosa con respecto a poder garantizar un mayor número de conexiones, en nuestra experiencia, a pesar de que algunos compañeros sí gozaban de una buena conexión, el enlentecimiento para el ingreso a las evaluaciones conllevaba a la reducción del tiempo para rendir algunas pruebas con cortos tiempos programados, lo que causaba su reprogramación o cancelación ocasional.
Con respecto al uso de la aplicación zoom para la supervisión de los exámenes, concordamos en que deberían explorarse el uso de nuevos aplicativos como los mencionados por el autor para la evaluación a distancia; o inclusive empezar a emplear nuevas metodologías como las pruebas a libro abierto ${ }^{(2)}$. Sin embargo, en nuestro mejor entendimiento, se sigue manteniendo la supervisión por medio de la plataforma Zoom, al menos, en la rendición de las evaluaciones parciales y finales de los cursos de pregrado, hasta el momento.

Para terminar, con respecto al cuestionario de Wong et al. ${ }^{(3)}$, aclaramos la intención de utilizar dicho cuestionario con el fin de evaluar las percepciones de docentes y alumnos sobre algunas herramientas tecnológicas, solo si es adaptado para este propósito tras su validación. A este respecto, en base a algunos estudios de percepción realizados en distintas carreras o escalas geográficas, consideramos pertinente poder contar con esta herramienta estandarizada ya que nos permitiría conocer la percepción de estudiantes y docentes de distintas profesiones en nuestro contexto a nivel local o nacional ${ }^{(4,5)}$.

La carta que hemos redactado muestra nuestra percepción como estudiantes de medicina de la Universidad Peruana Cayetano Heredia, Lima, Perú. Cada carrera o escuela de medicina de acuerdo a las características y experiencias previas de sus docentes y sus alumnos, así como la institución, van a tener preferencias diferentes que les ayudarán a crecer, desarrollar mejor las tecnologías de la información y comunicaciones. Esperamos de esta manera haber respondido y mejorado los puntos discordantes que ha podido observar en nuestra carta al editor.

Joel Joseph Gonzales Mendieta ${ }^{1,2, a}$, Fritz Fidel Váscones Román ${ }^{1,2, a}$, Ray Ticse ${ }^{2, b}$ 


\section{Correspondencia:}

Joel Joseph Gonzales Mendieta

Jr. Antonio Lishner 1798, Cercado, Lima, Perú.

Código postal 15082

joel.gonzales@upch.pe

Celular: +51953269663

\section{REFERENCIAS BIBLIOGRÁFICAS}

1. Plataformas virtuales de aprendizaje [Internet]. Lima: Universidad Peruana Cayetano Heredia; 2021 [Citado el 12 de octubre de 2021]. Disponible en: https://www.cayetano.edu.pe/cayetano/es/ noticias/1048-eva-blackboard

2. Zagury-Orly I, Durning SJ. Assessing openbook examination in medical education: The time is now. Med Teach. 2021 Aug; 43(8):972-973. doi: 10.1080/0142159X.2020.1811214
3. Wong G, Greenhalgh T, Pawson R. Internet-based medical education: a realist review of what works, for whom and in what circumstances. BMC Med Educ. 2010; 10:12. doi: 10.1186/1472-6920-10-12

4. Aguilar-Moncayo LN, García-Camacho GI, Morales-Rodas LY, Morales-Noriega SL. Percepción estudiantil acerca del uso de herramientas tradicionales vs tecnológicas, en el aprendizaje aplicado a las ciencias numéricas y lingüísticas. Pol Con. 2020 Mar; 43(5):192-210. doi: 10.23857/pc.v5i3.1331

5. Dost S, Hossain A, Shehab M, Abdelwahed A, AlNusair L. Perceptions of medical students towards online teaching during the COVID-19 pandemic: a national cross-sectional survey of 2721 UK medical students. BMJ Open. 2020 Nov 5; 10(11):e042378. doi: 10.1136/bmjopen-2020-042378

Recibido: 25/10/2021

Aceptado: 28/10/2021

1 Sociedad Científica de Estudiantes de Medicina Cayetano Heredia, Universidad Peruana Cayetano Heredia. Lima, Perú

2 Facultad de Medicina Alberto Hurtado, Universidad Peruana Cayetano Heredia. Lima, Perú.

a Estudiante de Medicina;

b Médico endocrinólogo 